\title{
Developpements Asymptotiques et Microfonctions dans les Classes de Gevrey
}

Par

\author{
JEAN-LOUIS ERMINE*
}

\section{§ I. Introduction}

Le travail qui suit se veut une étude "comparative" des développements asymptotiques et des microfonctions dans les classes de Gevrey.

Les outils employés dans l'un ou l'autre cas sont en dualité: fonctions Gevrey et ultradistributions, théorème de prolongement de Whitney et sa version duale etc... De plus, les méthodes utilisées dans les deux théories sont parfois très proches.

L'introduction des classes de Gevrey s'est révélée extrêmement fructueuse dans l'étude des équations différentielles à points singuliers irréguliers ([16] cf. théorème V. 8), de la resommation des solutions formelles d'une telle équation ([17]), des modules microdifférentiels ([13]). Ici, elle a, entre autre, l'intérêt de mettre sur un même plan l'étude des développements asymptotiques et des microfonctions (en effet, le cas classique de ceux-là est $s=+\infty$ et de celles-ci $s=1$ ).

Voici le plan de l'article. La première partie introduit les espaces fonctionnels utilisés par la suite.

La seconde partie ( $(\mathrm{IV})$ est consacrée aux développements asymptotiques. On généralise et on retrouve certains résultats de Malgrange [10] et Ramis [15] (corollaires IV. 6 et IV. 7).

La troisième partie $(\S \mathrm{V})$ donne la définition des microfonctions Gevrey de classe $s$ au point 0 de $C$. On établit un théorème d'indice dans les microfonctions Gevrey (théorème V. 6). Ces résultats sont ensuite appliqués pour montrer que dans une équation différentielle, toute solution formelle de classe de Gevrey $s$ converge, pour un ensemble

Communiqué par M. Kashiwara, le 24 décembre, 1984.

* Univertité de Bordeaux 1, U.E. R. de Mathématiques et d'Informatique, Laboratoire associé au CNRS $\mathrm{n}^{\circ} 226$ 351, cours de la Libération, 33405 TALENCE Cédex, France. 
de valeurs $s$ déterminé par l'irrégularité de l'équation (théorème $V .7$ ).

\section{§ II. Espaces Fonctionnels dans les Classes de Gevrey}

Soit $X$ une variété différentiable de dimension $n$, ou une variété différentiable à bord.

Soit $Y$ un fermé de $X$, ou $Y=\partial X$ [qui est localement un demiespace].

On supposera que $\partial Y$ est défini localement par l'équation $\rho(x)=0$ où $\rho$ vérifie

$$
|\rho(x)|>A d(x, \partial Y) \text {. }
$$

Dans la suite on travaillera sur un ouvert de coordonnées $U$ de $X$ tel que

$$
\partial Y \cap U=\{\rho=0\} .
$$

Soit $K$ un compact de $U$.

$\left.1^{\circ}\right)$ Fonctions et ultradistributions Gevrey de classe $s$ [8].

*) On note $\mathscr{G}_{s}(K, A)$ l'ensemble des fonctions $f, \mathscr{C}^{\infty}$ sur $K$ au sens de Whitney, qui vérifient la propriété suivante:

$$
\begin{gathered}
{ }^{\exists} c>0 \quad{ }^{\exists} A>0 \quad{ }^{\forall} \alpha \in \mathbb{N}^{n} \quad{ }^{\forall} x \in K \\
\left|D^{\alpha} f(x)\right|<c(\alpha !){ }^{s} A^{|\alpha|}
\end{gathered}
$$

On note $\mathscr{G}_{s}(K)=\underset{A>0}{\lim } \mathscr{G}_{s}(K, A)$.

L'ensemble des fonctions Gevrey (Roumieu) de classe $s$ sur $U$ est l'ensemble

$$
\mathscr{G}_{s}(U)=\lim _{\overleftarrow{K \subset U}} \lim _{A>0} \mathscr{G}_{s}(K, A)=\lim _{K \subset U} \mathscr{G}_{s}(K)
$$

*) On note $\mathscr{D}_{s}(K, A)$ l'ensemble des fonctions $\mathscr{C}^{\infty}$ de $X$, à support dans $K$, vérifiant la même propriété que les fonctions $\mathscr{G}_{s}(K, A)$.

On note $\mathscr{D}_{s}(U)=\underset{K \subset U}{\lim } \underset{A>0}{\lim } \mathscr{D}_{s}(K, A)$. C'est un espace DFN.

*) On appelle espace d'ultradistributions de classe $s$ sur un ouvert $U$ de $X$ l'ensemble ' $\mathscr{D}_{s}(U)$ dual fort de $\mathscr{D}_{s}(U)$. C'est un espace FN. 
*) $\mathscr{G}_{s}$ désigne le faisceau associé au préfaiseau

$$
U \rightarrow \mathscr{G}_{s}(U)
$$

C'est un faisceau fin. On a $\Gamma_{c}\left(U, \mathscr{G}_{s}\right)=\mathscr{D}_{s}(U)$.

$\mathscr{D}_{s}$ désigne le cofaisceau associé au copréfaisceau

$$
U \rightarrow \mathscr{D}_{s}(U)
$$

*) ' $\mathscr{D}_{s}$ désigne le faisceau associé au préfaisceau

$$
U \rightarrow^{\prime} \mathscr{D}_{s}(U) \text {. }
$$

$\mathrm{C}^{\prime}$ est un faisceau mou, mais pas flasque.

$2^{\circ}$ ) Fonctions à décroissance s-rapide et distributions s-tempérées.

*) On note $\mathscr{L}_{s, Y}(K, A, B)$ l'ensemble des fonctions $f$ de $\mathscr{G}_{s}(K, A)$ qui vérifient

$$
\begin{aligned}
{ }^{\exists} c>0 \quad{ }^{\forall} k \in \mathbb{N} \quad{ }^{\forall} x \in K-Y \quad{ }^{\forall} \alpha \in \mathbb{N}^{n} \\
\\
|\rho(x)|^{-k}\left|D^{\alpha} f(x)\right| \leq c A^{|\alpha|} B^{k}(a !)^{s}(k !)^{s-1}
\end{aligned}
$$

On note $\mathscr{L}_{s, Y}(U)=\lim _{\overleftarrow{K \subset U}} \lim _{\substack{A>0 \\ B>0}} \mathscr{L}_{s, Y}(K, A, B)$ c'est un espace complet de Schwartz.

Grâce à la formule de Taylor, on voit que $\mathscr{L}_{s, Y}(U)$ est l'ensemble des fonctions de $\mathscr{G}_{s}(U)$ infiniment plates sur $Y$.

*) On note $\mathscr{S}_{s, Y}(K, A, B)$ l'ensemble des fonctions de $\mathscr{D}_{s}(K, A)$ qui vérifient la même propriété que les fonctions de $\mathscr{L}_{s, Y}(K, A, B)$; et $\mathscr{S}_{s, Y}(U)=\lim _{\overline{K \subset U}} \underset{\substack{A>0 \\ B>0}}{\lim } \mathscr{S}_{s, Y}(K, A, B)$. C'est un espace DFS.

*) On appelle espace de distributions (ou ultradistributions) $s$ tempérées le long de $Y$, sur un ouvert $U$ de $X$, l'ensemble ' $\mathscr{S}_{s, Y}(U)$ dual fort de $\mathscr{S}_{s, Y}(U)$. C'est un espace FS. ' $\mathscr{S}_{s, Y}(U)$ s'identifie aux éléments de ' $\mathscr{D}_{s}(U-Y)$ qui sont prolongeables à $U$.

*) On note $\mathscr{L}_{s, Y}$ et ' $\mathscr{S}_{s, Y}$ les faisceaux associés correspondants. On a $\Gamma_{c}\left(U, \mathscr{L}_{s, Y}\right)=\mathscr{S}_{s, Y}(U), \Gamma_{c}\left(U,{ }^{\prime} \mathscr{S}_{s, Y}\right):==^{\prime} \mathscr{L}_{s, Y}(U)$ on note $\mathscr{S}_{s, Y}$ et ' $\mathscr{L}_{s, Y}$ les cofaisceaux associés correspondants. 
$3^{\circ}$ ) Théorème de Whitney.

*) On note $\mathscr{G}_{s, Y}(K, A)$ le sous-espace des fonctions de Whitney sur $Y F=\left(f_{\alpha}\right)_{\alpha \in N^{n}}$ qui vérifient:

1) ${ }^{\exists} c>0 \quad{ }^{\forall} \alpha \in \mathbb{N}^{n} \quad{ }^{\forall} x \in K \quad\left|f_{\alpha}(x)\right|<c A^{|\alpha|}(\alpha !)^{s}$.

2) En notant $R_{\alpha}^{m}(x, y)=f_{\alpha}(y)-\sum_{|\alpha+\beta| \leq m} f_{\alpha+\beta}(x) \frac{(y-x)^{\beta}}{\beta !}$

$$
\begin{gathered}
{ }^{\exists} c>0 \quad{ }^{\forall} \alpha,|\alpha| \leq m \quad{ }^{\forall} m \in \mathbb{N} \quad{ }^{\forall} x, y \in K \\
\left|R_{\alpha}^{m}(x, y)\right| \leq c|y-x|^{m-|\alpha|+1} A^{m+1}(m+1) !^{s} .
\end{gathered}
$$

Pour un ouvert $U$ de $X$, l'ensemble des fonctions Whitney Gevrey de classe sur $Y$ est l'ensemble

$$
\mathscr{G}_{s, Y}(U)=\lim _{K \subset U \cap Y} \underset{A>0}{\lim } \mathscr{G}_{s, Y}(K, A) .
$$

*) On note $\mathscr{D}_{s, Y}(K, A)$ le sous ensemble fermé de $\mathscr{G}_{s, Y}(K, A)$ des éléments dont l'extension $\mathscr{C}^{\infty}$ à $Y$ donnée par le théorème de Whitney est nulle en dehors de $K$.

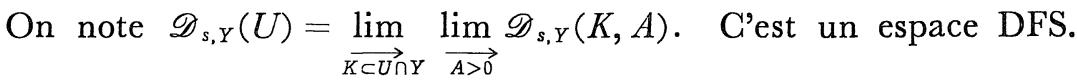

*) $\mathscr{G}_{s, Y}$ désigne le faisceau associé au préfaisceau

$$
U \rightarrow \mathscr{G}_{s, Y}(U) \text {. }
$$

On a $\Gamma_{c}\left(U, \mathscr{G}_{s, Y}\right)=\mathscr{D}_{s, Y}(U)$. $\mathscr{D}_{s, Y}$ désigne le cofaisceau associé.

*) Théorème de Whitney dans les Classes de Gevrey (cf. [6] par ex.). On a des suites exactes (avec morphismes continus)

$$
\begin{aligned}
& 0 \rightarrow \mathscr{L}_{s, Y}(U) \rightarrow \mathscr{G}_{s}(U) \rightarrow \mathscr{G}_{s, Y}(U) \rightarrow 0 \\
& 0 \rightarrow \Gamma_{Y}\left(U,^{\prime} \mathscr{D}_{s}\right) \rightarrow \Gamma\left(U,{ }^{\prime} \mathscr{D}_{s}\right) \rightarrow \Gamma\left(U,{ }^{\prime} \mathscr{S}_{s, Y}\right) \rightarrow 0
\end{aligned}
$$

\section{$\S$ III. Fonctions Holomorphes à Décroissance et à Croissance Exponentielle}

On suppose maintenant que $X-Y$ est une variété analytique complexe de dimension $1 . \bar{\partial}$ désigne la dérivation anti-holomorphe $\frac{\partial}{\partial \bar{z}}$ où $z$ est la coordonnée complexe de $X-Y$. 
$\left.1^{\circ}\right)$ Fonctions holomorphes à décroissance exponentielle d'ordre $t$ le long de $Y$.

Soit $f$ une fonction holomorphe sur $U-Y$. On dit qu'elle est à décroissance exponentielle d'ordre $t$ le long de $Y$ (ou a décroissance rapide d'ordre $t$ ) si elle vérifie

$$
{ }^{\vee} K \subset U \quad{ }^{\exists} c>0 \quad{ }^{\exists} T>0 \quad{ }^{\forall} x \in K-Y|f(x)| \leqq c \exp \left(-\frac{T}{|\rho(x)|^{t}}\right) .
$$

L'ensemble des fonctions holomorphes sur $U-Y$ à décroissance exponentielle d'ordre $t$ le long de $Y$ se note $\mathcal{O}_{t \text {-rap, } Y}(U)$ ou encore pour simplifier $\mathcal{O}_{t \text {-rap }}(U)$.

Proposition III.1. $f \in \mathcal{O}_{t-\mathrm{rap}}(U) \Leftrightarrow \bar{\partial} f=0 \quad$ et $\quad f \in \mathscr{L}_{S, Y}(U)$ avec $t=\frac{1}{s-1}$.

Démonstration. Il est aisé de voir que [cf. [4] p. 170 par exemple] que si $f \in \mathscr{L}_{s, Y}(U)$ alors ${ }^{{ }^{\vee}} \alpha \in N^{2} \quad{ }^{E} c_{\alpha}>0 \quad{ }^{\exists} T>0 \quad{ }^{{ }} x \in K-Y$ $\left|D^{\alpha} f(x)\right| \leq c_{\alpha} \exp \left(-\frac{T}{|\rho(x)|^{t}}\right)$. Si de plus $\frac{\partial f}{\partial \bar{z}}=0$, ceci prouve que $f \in$ $\mathcal{O}_{t \text {-rap }}(U)$. Réciproquement si $f \in \mathcal{O}_{t-\text { rap }}(U)$ en appliquant la formule de Cauchy dans $U-Y$ à $f$ sur un disque de centre $x$ et de rayon $\lambda|\rho(x)|(\lambda$ assez petit)

$$
\left|D^{\alpha} f(x)\right| \leq c \alpha !|\lambda \rho(x)|^{-|\alpha|} \sup _{|\zeta-x|=\lambda|\rho(x)|} \exp \left(-\frac{T}{|\rho(\zeta)|^{t}}\right) .
$$

Pour $|\zeta-x|=\lambda|\rho(x)|$ et grâce à la condition imposée à $\rho$ on a

$$
|\rho(\zeta)|<c_{1} d(\zeta, Y)<c_{1}[|\zeta-x|+d(x, Y)]<c^{\prime}|\rho(x)|
$$

d'où

$$
\left|D^{\alpha} f(x)\right|<c_{\alpha}|\rho(x)|^{-\alpha} \exp \left(-\frac{T}{|\rho(x)|^{t}}\right)
$$

en utilisant l'inégalité obtenue par dérivation

$$
x^{-j} \exp \left(-\frac{\varepsilon}{x^{r}}\right) \leq\left(\frac{j}{\varepsilon t}\right)^{1 / t}
$$

on obtient $\left|D^{\alpha} f(x)\right| \leqq c_{\alpha}^{\prime} \exp \left(-\frac{T^{\prime \prime}}{|\rho(x)|^{t}}\right)$ d'où le résultat.

$2^{\circ}$ ) Fonctions holomorphes à croissance exponentielle d'ordre $t$ le long 
de $Y$.

Soit $f$ une fonction holomorphe sur $U-Y$. On dit qu'elle est à croissance exponentielle d'ordre $t$ le long de $Y$ (ou a croissance modérée d'ordre $t$ ) si

$$
{ }^{\forall} K \subset U{ }^{\forall} \eta>0{ }^{\exists} c>0 \quad{ }^{\forall} x \in K-Y|f(x)| \leq c \exp \left(\frac{\eta}{|\rho(x)|{ }^{t}}\right) .
$$

L'ensemble des fonctions holomorphes sur $U-Y$ à croissance exponentielle d'ordre $t$ le long de $Y$ se note $\mathcal{O}_{t-\text { mod, } Y}(U)$ ou encore pour simplifier $\mathcal{O}_{t-\text { mod }}(U)$.

Proposition III. 2. $f \in \mathcal{O}_{t-m o d}(U) \Leftrightarrow \bar{\partial} f=0$ et $f \in^{\prime} \mathscr{S}_{s, Y}(U)$ avec $t=\frac{1}{s-1}$.

cf. par exemple [4] p. 137 ou [19] pour la dimension 1 [20] ou[21] pour la dimension $n$.

\section{§ IV. Développements Asymptotiques dans les Classes de Gevrey}

\section{$\left.1^{\circ}\right)$ Définition.}

Soit $V$ un secteur angulaire ouvert de $\boldsymbol{C}$ de sommet 0 . Soit $f$ une fonction holomorphe dans $V$ et soit $\hat{f}=\sum_{p=0}^{+\infty} a_{p} z^{p}$ une série formelle soit $s$ un réel supérieur à 1 .

On dit que $f$ admet $\hat{f}$ pour développement asymptotique Gevrey de classe $s$ en 0 si pour tout sous-secteur fermé $\bar{W}$ de $V$, il existe des constantes $C_{W}$ et $A_{W}$ telles que

$$
{ }^{\forall} k \in N{ }^{\forall} z \in \bar{W}\left|f(x)-\sum_{p=0}^{k} a_{p} z^{p}\right|<C_{W} A_{W}^{k}(k !)^{s-1}|z|^{k} .
$$

L'ensemble des fonctions $f$ admettant un développement asymptotique Gevrey de classe $s$ dans $V$ note $A_{s}(V)$.

On note $A_{0, s}(V)$ l'ensemble des fonctions admettant un développement asymptotique nul dans $V$.

Si $f \in A_{0, s}(V)$ alors on vérifie aisément que pour tout fermé $\bar{W}$ de $V$, il existe des constantes $C_{W}$ et $a_{W}$ tel que 


$$
{ }^{\forall} z \in \bar{W}|f(z)|<c_{W} \exp \left(-\frac{a_{W}}{|z|^{t}}\right) \text { où } t=1 / s-1
$$

et réciproquement.

\section{$2^{\circ}$ ) Faisceau des développements asymptotiques.}

On peut interpréter les développements asymptotiques comme un faisceau sur l'éclaté réel de 0 dans $\mathbb{C}$.

Soit $S^{1}$ l'ensemble des nombres complexes de module 1. On note $\tilde{\boldsymbol{C}}$ le sous ensemble de $\boldsymbol{C} \times S^{1}$ suivant:

$$
\tilde{C}=\left\{\left(z, \frac{z}{|z|}\right) \text { si } z \neq 0 \quad(0, \xi) \quad{ }^{\forall} \xi \in S^{1}\right\} .
$$

On munit $\tilde{\boldsymbol{C}}$ de la topologie induite (un voisinage d'un point $\xi$ de $S^{1}$ est un arc de cercle $\tilde{V}(\xi)$ contenant $\xi$, il est intercepté par un secteur angulaire de sommet 0 qu'on notera $V(\xi))$. On note $S=$ $\{0\} \times S^{1}$.

Il est clair que $\tilde{\boldsymbol{C}}-S$ est homéomorphe à $\boldsymbol{C}-\{0\}$, et au voisinage d'un point $\left(0, \xi_{0}\right)$, la carte locale donnée par les coordonnées polaires:

$$
(z, \xi) \rightarrow(\operatorname{Arg} \xi,|z|)=(\theta, r)
$$

munit $\tilde{\boldsymbol{C}}$ d'une structure de variété à bord, de bord $S$.

On peut donc parler de fonctions différentiables, Gevrey de classe $s$ etc... sur $\tilde{\boldsymbol{C}}$, qu'on notera $\tilde{\mathscr{E}}, \widetilde{\mathscr{G}}_{s}$ etc....

On note $\tau$ la projection naturelle $\tilde{\boldsymbol{C}} \rightarrow \boldsymbol{C}$.

On définit le faisceau $\mathscr{A}_{0, s}$ sur $\tilde{\boldsymbol{C}}$ (et de même le faisceau $\mathscr{A}_{s}$ ) par:

$$
\mathscr{A}_{0, s \mid \tilde{C}-s}=\mathcal{O}_{c-\{0\}} \text {. }
$$

Si $V$ est un ouvert de $S$, auquel il correspond un secteur angulaire dans $C$ noté $V$

$$
\mathscr{A}_{0, s \mid S}(\tilde{V})=\underset{U}{\lim } A_{0, s}(V \cap U)
$$

où $U$ parcourt un système fondamental de voisinages de 0 dans $\mathbb{C}$

$3^{\circ}$ ) Résolution du faisceau $\mathscr{A}_{0, s}$.

Soit $\widetilde{\mathscr{L}}_{s, s}$ le faisceau sur $\tilde{C}$ des fonctions de $\widetilde{\mathscr{G}}$ plates sur $S$ (qui sont aussi les fonctions à décroissance $s$-rapide le long de $S$ ).

$\tilde{\mathscr{L}}_{s, s}$ est un faisceau fin (puisque c'est un $\widetilde{\mathscr{G}}_{s}$-module) et on a: 


$$
\tilde{\mathscr{L}}_{s, S}(S)=\left(\mathscr{L}_{s,(0\})}\right)_{0} \text { et } \tilde{\mathscr{L}}_{s, s}(\boldsymbol{C})=\mathscr{L}_{s,\{0\}}(\boldsymbol{C})
$$

Proposition IV.1. On a une suite exacte de faisceaux

$$
0 \rightarrow \mathscr{A}_{0, s} \longrightarrow \widetilde{\mathscr{L}}_{s, s} \stackrel{\tilde{\partial}}{\longrightarrow} \tilde{\mathscr{L}}_{s, s} \longrightarrow 0 .
$$

Démonstration [cf. 10].

*) Pour les fibres aux points de $\tilde{\boldsymbol{C}}-S$, ce n'est autre que la résolution du $\bar{\partial}$ dans $\mathscr{G}_{s}$ car $\mathscr{A}_{0, s \mid \tilde{C}-s}=\mathcal{O}_{c-(0)}$ et $\tilde{\mathscr{L}}_{s, S \mid \tilde{C}-s}=\mathscr{G}_{s \mid c-(0)}$, qu'on obtient ici par la résolution $\mathscr{C}^{\infty}$ habituelle et l'ellipticité de $\bar{\partial}$ en degré 0 .

Montrons maintenant le résultat pour les faisceaux restreints à $S$.

*) De par la remarque faite au IV $1^{\circ}$ ), on voit que $\mathscr{A}_{0, s}=\mathcal{O}_{t \text {-rap. } s}$ avec $t=\frac{1}{s-1}$, donc le noyau de l'application $\widetilde{\mathscr{L}}_{s, s} \stackrel{\tilde{\partial}}{\longrightarrow} \widetilde{\mathscr{L}}_{s, s}$ est bien $\mathscr{A}_{0, s}$ d'après la proposition III. 1 .

*) Soit $\tilde{V}$ un fermé de $S$, soit $f \in \tilde{\mathscr{L}}_{s, S \mid S}(\tilde{V})=\underset{\vec{U}}{\lim } \mathscr{L}_{s,\{0\}}(V \cap U)$ notons encore $f$ le représentant dans $\mathscr{L}_{s,(0)}(V)$. Par finesse, $f$ se prolonge en une section $g$ de $\mathscr{L}_{s,\{0\}}(\boldsymbol{C})$. Soit $h$ une fonction Gevrey de classe $s$ qui vérifie $\frac{\partial h}{\partial \bar{z}}=g$.

La série de Taylor de $h$ en 0 est une série formelle $h$ vérifiant les conditions de croissance Gevrey de classe $s$, de plus elle ne contient pas de terme en $\bar{z}$, i.e:

$$
h \in \boldsymbol{C}[[z]]_{s}=\left\{\sum_{n \in \boldsymbol{N}} a_{n} z^{n} \in \boldsymbol{C}[[z]] \sum_{n \in \boldsymbol{N}} \frac{a_{n}}{(n !)^{s-1}} z^{n} \in \boldsymbol{C}\{z\}\right\} .
$$

D'après théorème de Borel-Ritt Gevrey ([17]), si $V$ est assez petit, il existe $h_{1} \in A_{s}(V)$ tel que $h_{1}$ admette $\hat{h}$ comme développement asymptotique. Posons alors $h-h_{1}=k, k$ est un élément de $\mathscr{G}_{s}(v)$ infiniment plat en 0 et comme $\bar{\partial} h_{1}=0$ elle vérifie $\frac{\partial k}{\partial \bar{z}}=\left.g\right|_{V}=f$, ce qui montre la surjectivité du $\bar{\partial}$.

$\left.4^{\circ}\right)$ L'isomorphisme de Malgrange-Ramis ([10] [15]). 
Grâce au théorème de Whitney, on a une suite exacte complexes de faisceaux mous:

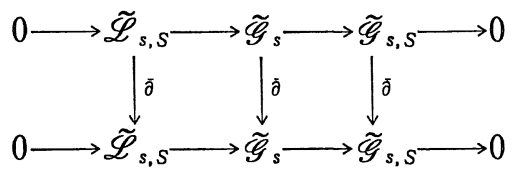

En prenant les sections sur $S$ de cette suite exacte on obtient une autre suite exacte d'espaces vectoriels

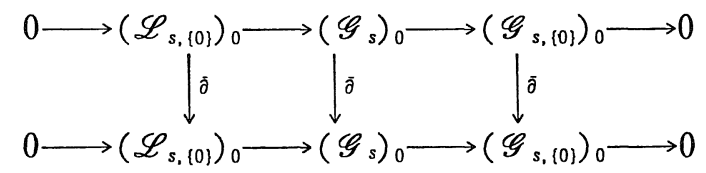

On écrit ensuite la longue suite exacte de cohomologie associée, d'après le résultat précédent on a

$$
\begin{aligned}
& \operatorname{Ker}\left(\left(\mathscr{L}_{s,\{0\}}\right)_{0} \stackrel{\tilde{\partial}}{\longrightarrow}\left(\mathscr{L}_{s,\{0\}}\right)_{0}\right)=\mathscr{A}_{0, s}(S)=0 \\
& \operatorname{coker}\left(\left(\mathscr{L}_{s,\{0\}}\right)_{0} \stackrel{\bar{\partial}}{\longrightarrow}\left(\mathscr{L}_{s,\{0\}}\right)_{0}\right)=H^{1}\left(S, \mathscr{A}_{0, s}\right) .
\end{aligned}
$$

Il est de plus clair que

$$
\begin{aligned}
& \operatorname{Ker}\left(\left(\mathscr{G}_{s}\right)_{0} \stackrel{\bar{\partial}}{\longrightarrow}\left(\mathscr{G}_{s}\right)_{0}\right)=\boldsymbol{C}\{z\} \\
& \operatorname{coker}\left(\left(\mathscr{G}_{s}\right)_{0} \stackrel{\bar{\partial}}{\longrightarrow}\left(\mathscr{G}_{s}\right)_{0}\right)=0 \\
& \operatorname{Ker}\left(\left(\mathscr{G}_{s, 00\}}\right)_{0} \stackrel{\bar{\partial}}{\longrightarrow}\left(\mathscr{G}_{s,(00)}\right)_{0}\right)=\mathbb{C}[[z]]_{s} .
\end{aligned}
$$

On obtient donc:

Theoreme IV.2. Il y a un isomorphisme

$$
\boldsymbol{C}[[z]]_{s} / \boldsymbol{C}\{z\} \stackrel{\sim}{\longrightarrow} H^{1}\left(S, \mathscr{A}_{0, s}\right) .
$$

$\left.5^{\circ}\right)$ Généralisation d'un théorème de Malgrange [10].

Sur $\boldsymbol{C}$ on considère le faisceau $\mathscr{M}_{0, s}$ des fonctions holomorphes sur $\boldsymbol{C}-\{0\}$ vérifiant la propriété suivante:

Si $U$ est un voisinage de 0

$$
\begin{aligned}
& \mathscr{M}_{0, s}(U)=\left\{f \in \mathcal{O}(U-\{0\}) f(z)=\sum_{n \in Z} a_{n} z^{n} \text { avec } \sum_{n>0} a_{-n}(n !)^{s-1} T^{n}=g(T)\right. \\
& \text { est une fonction entière }\} .
\end{aligned}
$$


Pour $s=+\infty, \mathscr{M}_{0,+\infty}$ désigne le faisceau des fonctions méromorphes de pôle 0 .

Soit $P$ un opérateur différentiel d'ordre fini à coefficients analytiques au voisinage de 0 .

On note $L \cdot$ le complexe de faisceaux sur $\tilde{C}$ suivant:

$$
L: \mathscr{A}_{0, s} \stackrel{P}{\longrightarrow} \mathscr{A}_{0, s}
$$

on note $\mathscr{A}_{0, s, P}=H^{0}\left(L^{*}\right)=\operatorname{Ker} P$.

On considère le complexe de faisceaux sur $\boldsymbol{C}$ :

$$
M \cdot: \mathscr{M}_{0, s} \stackrel{p^{*}}{\longrightarrow} \mathscr{M}_{0, s}
$$

On s'intéresse aux faisceaux des "solutions" (cf. par ex [14]) de $P^{*}$ dans $\mathscr{M}_{0, s}: \operatorname{Ker} P^{*}$ et coker $P^{*}$, qui sont les groupes de cohomologie de $M$.

On a alors le résultat suivant:

Theoreme IV.3. Le dual topologique de $H^{i}\left(\boldsymbol{C}, M^{*}\right)$ est isomorphe $\grave{a} H_{c}^{2-i}\left(\tilde{\boldsymbol{C}}, L^{\cdot}\right)$.

Pour démontrer ce théorème nous aurons besoin des lemmes cidessous:

Lemme IV.4. On a une suite exacte de faisceaux

$$
0 \longrightarrow \mathscr{M}_{0, s} \longrightarrow \mathscr{S}_{s,\{0\}} \stackrel{\bar{\partial}}{\longrightarrow} \mathscr{S}_{s,\{0\}} \longrightarrow 0 .
$$

Démonstration. On a un isomorphisme (de module différentiel):

$$
\mathscr{M}_{0, s}=\mathscr{O} \oplus \frac{\Gamma_{\{0\}}\left(\mathscr{D}_{s}\right)}{\tilde{\partial} \underline{\Gamma}_{\{0\}}\left(\mathscr{D}_{s}\right)} .
$$

En effet, on sait [8] que toute ultradistribution sur $\boldsymbol{C}$ à support dans $\{0\}$ est une somme de dérivées de Dirac à l'origine. Plus précisément si $T \in \Gamma_{\{0\}}\left(\mathscr{D}_{\mathrm{s}}\right)$

$$
\begin{aligned}
& T=\sum_{\alpha \in N^{2}} a_{\alpha} D^{\alpha} \delta(z, \bar{z}) \text { avec la condition } \\
& { }^{\forall} A>0 \quad{ }^{\Xi_{C}}>0 \quad\left|a_{\alpha}\right|<c \frac{A^{|\alpha|}}{(\alpha !)^{s}} .
\end{aligned}
$$

Ainsi un élément de $\frac{\Gamma_{\{0\}}\left({ }^{\prime} \mathscr{D}_{s}\right)}{\bar{\partial} \underline{\Gamma}_{[0\}}\left({ }^{\prime} \mathscr{D}_{s}\right)}$ se représente d'une manière unique 
par

$$
\begin{aligned}
& \dot{T}=\sum_{n \in N} a_{n} \delta^{(n)}(z) \text { avec la condition } \\
& { }^{\forall} A>0 \quad{ }^{\exists} c>0 \quad\left|a_{n}\right|<c \frac{A^{n}}{(n !)^{s}} .
\end{aligned}
$$

Cet élément est identifié isomorphiquement à un élément de l'ensemble $\left\{\sum_{n<0} a_{n} z^{n}\right.$ avec $\sum a_{-n}(n !)^{s-1} T^{n}=g(T)$ est une fonction entière $\}$ grâce à l'application (déduite de l'application valeur principale)

$$
\sum_{n \in N} \frac{a_{n}}{z^{n-1}} \rightarrow 2 i \pi \sum_{n \in N} \frac{(-1)^{n}}{n !} a_{n} \delta^{(n)}(z)
$$

D'où l'isomorphisme en question.

Ensuite, on écrit la suite exacte de complexes de faisceaux (mous) donnée par le théorème de Whitney:

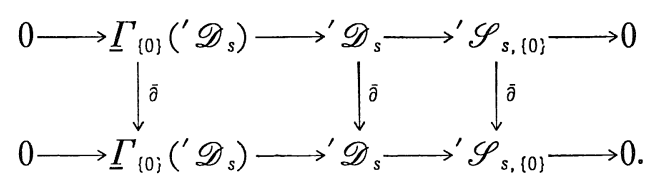

On applique la longue suite exacte de cohomologie, et on obtient le lemme IV. 4.

Lemme IV.5. Le complexe $M^{\cdot}(\boldsymbol{C})$ est à cohomologie de dimension finie.

Démonstration. Ceci résulte des théorèmes d'indices de [11] et [16].

La caractéristique d'Euler-Poincaré de $M^{*}(\mathbb{C})$ (i. e. l'indice de $P^{*}$ dans $\left.\mathscr{M}_{0, s}(\boldsymbol{C})\right)$ est égale à $\chi_{1}\left(P^{*}\right)-\chi_{s}\left(P^{*}\right)$ où $\chi_{1}\left(P^{*}\right)$ est l'indice de $P^{*}$ dans $\boldsymbol{C}\{z\}$ et $\chi_{s}\left(P^{*}\right)$ l'indice de $P^{*}$ dans $\mathbb{C}[[z]]_{\text {s。 }}$

Démonstration du théorème. Grâce au lemme IV. 4, le faisceau ' $\mathscr{S}_{s,\{0\}}$ étant fin, la cohomologie de $M^{\circ}(\boldsymbol{C})$ est égale à la cohomologie du complexe simple associé au complexe double

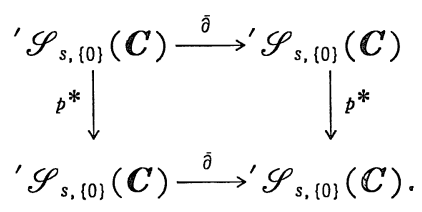

Cette cohomologie étant de dimension finie par la proposition pré- 
cédente, les espaces étant de type FS, on peut appliquer le lemme de dualité ([1] par exemple) et le dual des groupes de cohomologie ci-dessus est égal aux groupes de cohomologie (de degré $2-j$ ) du complexe simple associé au complexe double suivant:

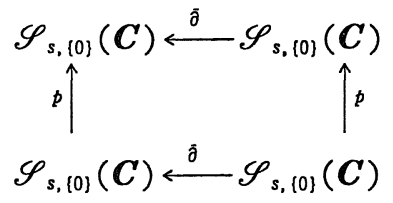

qui n'est autre que le complexe double suivant:

$$
\begin{aligned}
& \tilde{\mathscr{S}}_{s, S}(\tilde{\boldsymbol{C}})=\Gamma_{c}\left(\tilde{\boldsymbol{C}}, \tilde{\mathscr{L}}_{s, S} \stackrel{\tilde{\sigma}}{\longleftarrow} \Gamma_{c}\left(\tilde{\boldsymbol{C}}, \tilde{\mathscr{L}}_{s, S}\right)=\tilde{\mathscr{S}}_{s, S}(\tilde{\boldsymbol{C}})\right. \\
& p \uparrow \quad \uparrow p \\
& \Gamma_{c}\left(\tilde{\boldsymbol{C}}, \tilde{\mathscr{L}}_{s, s}\right) \stackrel{\bar{\jmath}}{\longleftarrow} \Gamma_{c}\left(\tilde{\boldsymbol{C}}, \tilde{\mathscr{L}}_{s, S}\right)
\end{aligned}
$$

Les faisceaux $\tilde{\mathscr{L}}_{s, s}$ étant fins donc c-mous, on obtient le résultat grâce à la proposition IV. 1 .

Corollaire IV.6. Le dual topologique de $H^{i}\left(\boldsymbol{C}, M^{*}\right)$ est isomorphe à $H_{c}^{2-i}\left(\tilde{C}, \mathscr{A}_{0, s, P}\right)$.

Démonstration. Il suffit d'appliquer au résultat précédent la suite exacte de faisceaux:

$$
0 \longrightarrow \mathscr{A}_{0, s, P} \longrightarrow \mathscr{A}_{0, s} \stackrel{P}{\longrightarrow} \mathscr{A}_{0, s} \longrightarrow 0
$$

qui est théorème fondamental des développements asymptotiques énoncé dans [15].

Corollaire IV.7. $\operatorname{Dim}_{\boldsymbol{c}} H_{c}^{1}\left(\widetilde{\boldsymbol{C}}, \mathscr{A}_{0, s, P}\right)=\chi_{s}(P)-\chi_{1}(P)$.

Démonstration. Elle est immédiate grâce au théorème IV. 3. et au lemme IV. 5 et du fait que $\chi\left(P^{*}\right)=-\chi(P)$.

On retrouve ainsi un théorème de Ramis énoncé dans [15].

\section{§ V. Microfonctions dans les Classes de Gevrey}

$\left.1^{\circ}\right)$ Co-éclaté $\boldsymbol{C}$ en 0.

Si $V$ est un secteur ouvert de $\boldsymbol{C}$ d'origine 0 , on note $V^{\perp}$ le polaire 
de $V$ et $V^{*}$ le secteur dual de $V$

$$
\begin{aligned}
& V^{\perp}=\left\{\left.\zeta \in C\right|^{\forall} \xi \in V\langle\xi, \zeta\rangle \geq 0\right\} \\
& V^{*}=\operatorname{Int}\left(V^{\perp}\right) .
\end{aligned}
$$

On note $S^{1 *}$ le cercle des nombres complexes de module 1 muni de la topologie duale de $S^{1}$, c'est-à-dire les ouverts de $S^{1 *}, \tilde{V}^{*}$ sont interceptés par des secteurs angulaires $V^{*}$ duaux des secteurs $V$ interceptant des ouverts $\tilde{V}$ de $S^{1}$. (En fait $S^{1 *}$ est homéomorphe à $\left.S^{1}\right)$.

On note $\tilde{C}^{*}$ le sous-ensemble de $\boldsymbol{C} \times S^{1 *}$ suivant:

$$
\tilde{C}^{*}=\left\{\left(z, \frac{z}{|z|}\right) \text { si } z \neq 0 ;(0 ; \zeta) \quad{ }^{\forall} \zeta \in S^{1 *}\right\} .
$$

On munit $\tilde{C}^{*}$ de la topologie ainsi définie:

On note $D$ le sous-ensemble de $S^{1} \times S^{1 *}$ défini par

$$
D=\{(\xi, \zeta)<\xi, \zeta>\geq 0\} .
$$

On considère $\tilde{C}^{+}$le sous-ensemble de $\boldsymbol{C} \times S^{1} \times S^{1 *}$ ainsi défini

$$
\tilde{C}^{+}=\left\{\left(z, \frac{z}{|z|}, \frac{z}{|z|}\right) \text { si } z \neq 0(0, \xi, \zeta)^{\forall}(\xi, \zeta) \in D\right\} .
$$

On munit $\tilde{C}^{+}$de la topologie suivante (non séparée)

-Si $z_{0} \neq 0$, un voisinage de $\left(z_{0}, \frac{z_{0}}{\left|z_{0}\right|}\right)$ est l'ensemble des points $z$ tels que $z$ appartient à un voisinage de $z_{0}$ dans $\boldsymbol{C}-\{0\}$

$-\mathrm{Si} z_{0}=0$ un voisinage de $\left(0, \xi_{0}, \zeta_{0}\right)$ est le sous ensemble de $V(0) \times \tilde{V}\left(\xi_{0}\right) \times V^{*}\left(\zeta_{0}\right) \cap \tilde{C}^{+}$des points $(z, \xi, \zeta)$ tels que

Si $z=0 \quad \xi \in \tilde{V}\left(\xi_{0}\right) \quad \zeta \in \tilde{V}^{*}\left(\zeta_{0}\right) \quad$ avec $\quad\langle\xi, \zeta>\geq 0$

Si $z \neq 0 \quad \xi=\zeta=\frac{z}{|z|} \quad$ avec $\quad \xi \in V\left(\xi_{0}\right)$.

On a des projections:

$$
\begin{array}{ll}
\pi^{\prime}: \tilde{C}^{+} \longrightarrow \tilde{C} \\
\tau^{\prime}: \tilde{C}^{+} \longrightarrow \tilde{C}^{*}
\end{array} \text { et } \pi: C^{*} \longrightarrow C .
$$

On munit $\tilde{C}^{*}$ de la topologie quotient associée à $\tau^{\prime}$ [i.e. pour la relation d'équivalence: $\left.(z, \xi, \zeta) \sim\left(z^{\prime}, \xi^{\prime}, \zeta^{\prime}\right) \Leftrightarrow z=z^{\prime}, \zeta=\zeta^{\prime}\right]$.

Cette topologie se décrit ainsi:

-Si $z_{0} \neq 0$ un voisinage de $\left(z_{0}, \frac{z_{0}}{\left|z_{0}\right|}\right)$ est l'ensemble des points 
$\left(z, \frac{z}{\left|z_{0}\right|}\right)$ tels que $z$ appartient à un voisinage de $z_{0}$ dans $\boldsymbol{C}-\{0\}$.

-Si $z_{0}=0$ un voisinage de $\left(0, \zeta_{0}\right)$ est le sous-ensemble de $V(0) \times \tilde{V}^{*}(\zeta) \cap \tilde{C}^{*}$ des points $(z, \zeta)$ tels que

Si $z=0 \zeta \in \tilde{V}^{*}\left(\zeta_{0}\right)$

Si $z \neq 0 \quad \zeta=\frac{z}{|z|}$ avec $\zeta \in \tilde{V}^{*}(\xi), \xi$ est tel qu'il existe $\zeta \in \tilde{V}^{*}\left(\zeta_{0}\right)$ tel que $\langle\xi, \zeta\rangle \geq 0$.

On a alors un diagramme commutatif d'applications continues

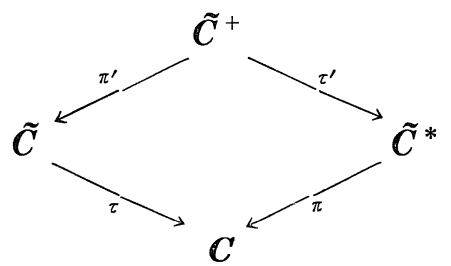

On note $S^{*}=\{0\} \times S^{1 *}=\pi^{-1}(\{0\})$.

Cette topologie est assez pathologique, sauf sur $S^{*}$, où elle coïncide avec celle de $S$. Cependant, il est "équivalent" d'étudier un faisceau sur $\tilde{\boldsymbol{C}}$ ou $\tilde{\boldsymbol{C}}^{*}$, ainsi que sa cohomologie, ou sa cohomologie locale dans $S^{*}$, comme le montrent les propositions suivantes [18].

Proposition V.1. 1) Soit $\mathscr{F}$ un objet de la catégorie dérivée des faisceaux sur $\boldsymbol{C}$, on a un isomorphisme

$$
\begin{aligned}
& \boldsymbol{R} \tau_{*}^{\prime} \pi^{\prime-1} \boldsymbol{R} \Gamma_{S}\left(\tau^{-1} \mathscr{F}\right) \rightarrow \boldsymbol{R} \Gamma_{s^{*}}\left(\pi^{-1} \mathscr{F}\right) \\
& \boldsymbol{R} \tau_{*} \boldsymbol{R} \Gamma_{S}\left(\tau^{-1} \mathscr{F}\right) \rightarrow \boldsymbol{R} \pi_{*} \boldsymbol{R} \Gamma_{s^{*}}\left(\pi^{-1} \mathscr{F}\right) .
\end{aligned}
$$

2) Les catégories dérivées des faisceaux abéliens $\mathscr{F}$ sur $\tilde{\boldsymbol{C}}$ et $\mathscr{G}$ sur $\boldsymbol{C}^{*}$ sont équivalentes, on a l'isomorphisme

$$
\mathscr{G}=\boldsymbol{R} \tau_{*}^{\prime} \pi^{\prime-1} \mathscr{F} \text {. }
$$

Enonçons aussi la proposition suivante [18]

Proposition V.2. Soit $\mathscr{F}$ un faisceau sur $\boldsymbol{C}$, pour tout point $(0, \zeta)$ de $S^{*}$ on $a\left(\zeta=e^{i \theta}\right)$

$$
\left(\underline{H}_{s^{*}}^{p}\left(\pi^{-1} \mathscr{F}\right)\right)_{(0, \zeta)}=\lim _{\longrightarrow} H_{U \cap V}^{p}(U, \mathscr{F})
$$

où $U$ et $V$ décrivent (par exemple) la famille 


$$
U_{\varepsilon}=\{z \in \boldsymbol{C}|| z \mid<\varepsilon\} \quad V_{\varepsilon, \zeta}=\left\{z|\operatorname{Arg} z-\theta| \leq \frac{\pi}{2}+\varepsilon\right\}
$$

$2^{\circ}$ ) Microfonctions.

Soit la suite exacte (Résolution de $\mathcal{O}$ par les ultradistributions)

$$
0 \longrightarrow \mathcal{O} \longrightarrow \mathscr{D}_{s} \stackrel{\bar{\partial}}{\longrightarrow} \mathscr{D}_{s} \longrightarrow 0\left(\bar{\partial}=\frac{\partial}{\partial \bar{z}}\right) .
$$

L'exactitude de cette suite est simple à établir en dimension un; il suffit par exemple de résoudre le $\bar{\partial}$ dans les hyperfonctions et d'utiliser l'ellipticité du $\bar{\partial}$.

On applique ensuite à cette suite exacte le foncteur $\pi^{-1}$, qui est exact, puis le foncteur $\underline{\Gamma}_{s^{*}}$ qui lui n'est plus exact, on obtient une suite exacte longue de faisceaux sur $\tilde{C}^{*}$

$$
0 \longrightarrow \underline{\Gamma}_{s^{*}}\left(\pi^{-1} \mathcal{O}\right) \longrightarrow \underline{\Gamma}_{s^{*}}\left(\pi^{-1} \mathscr{D}_{s}\right) \longrightarrow \underline{\Gamma}_{s^{*}}\left(\pi^{-1}, \mathscr{D}_{s}\right) \longrightarrow \cdots
$$

D'après la proposition V.2, $\underline{\Gamma}_{s^{*}}\left(\pi^{-1} \mathcal{O}\right)$ est réduit à $\{0\}$. On note

$$
\mathscr{C}_{0}^{\boldsymbol{R}, s}=\operatorname{coker}\left(\underline{\Gamma}_{s^{*}}\left(\pi^{-1} \mathscr{D}_{s}\right) \stackrel{\bar{\partial}}{\longrightarrow} \underline{\Gamma}_{s^{*}}\left(\pi^{-1} \mathscr{D}_{s}\right)\right) \text { et on a }
$$

Proposition et Definition V. 3. On a une suite exacte de faisceaux sur $\tilde{C}^{*}$

$$
0 \longrightarrow \underline{\Gamma}_{s^{*}}\left(\pi^{-1} \mathscr{D}_{s}\right) \stackrel{\bar{\partial}}{\longrightarrow} \underline{\Gamma}_{s^{*}}\left(\pi^{-1}{ }^{\prime} \mathscr{D}_{s}\right) \longrightarrow \mathscr{C}_{0}^{R, s} \longrightarrow 0
$$

$\mathscr{C}_{0}^{\boldsymbol{R}, \boldsymbol{s}} \mathrm{s}^{\prime}$ appelle le faisceau des microfonctions de classe $s$ en 0.

Remarque. Dans [18] on définit les microfonctions en prenant en plus l'image inverse des faisceaux $\pi^{-1} \mathscr{D}_{s}$ par l'application antipodale de $S^{*}$; nous ne l'avons pas fait ici.

$3^{\circ}$ ) Image directe des microfonctions.

Soit $\mathscr{C}_{s}$ le faisceau des opérateurs ultradifférentiels à coefficients constants sur $\boldsymbol{C}([8])$ c'est-à-dire, en notant $\partial=\frac{d}{d z}$

$$
\mathscr{C}_{s}=\left\{P=\sum_{n \in N} a_{n} \partial^{n}:{ }^{\forall} A>0 \quad{ }^{{ }} c>0 \quad\left|a_{n}\right| \leq c \frac{A^{n}}{(n !)^{s}}\right\}
$$


Si $P \in \mathscr{C}_{s}, \delta$ désignant la distribution de Dirac à l'origine, on pose formellement

$$
P \delta=\sum_{n \in N} a_{n} \delta^{(n)} .
$$

On note $\mathscr{U}_{s}=\mathscr{C}_{s} \delta$ (cf. [16]).

Theoreme V.4. On a une suite exacte de faisceaux (de $\mathcal{O}$-modules) sur C

$$
0 \longrightarrow \mathscr{U}_{s} \longrightarrow \pi_{*} \mathscr{C}_{0}^{R, s} \longrightarrow \mathcal{O}_{c \mid\{0\}} \longrightarrow 0
$$

De plus $R^{i} \pi_{*} \mathscr{C}_{0}^{\boldsymbol{R}, s}=0$ si $i>0$.

On utilise ici la suite exacte

$$
0 \longrightarrow \underline{\Gamma}_{s^{*}}\left(\pi^{-1}{ }^{\prime} \mathscr{D}_{s}\right) \stackrel{\bar{\partial}}{\longrightarrow} \underline{\Gamma}_{s^{*}}\left(\pi^{-1} \mathscr{D}_{s}\right) \longrightarrow \mathscr{C}_{0}^{n, s} \longrightarrow 0 .
$$

En écrivant la longue suite exacte de cohomologie, on obtient:

$$
\begin{aligned}
0 \rightarrow \pi_{*} \underline{\Gamma}_{s^{*}}\left(\pi^{-1} \mathscr{D}_{s}\right) \rightarrow \pi_{*} \underline{\Gamma}_{s^{*}}\left(\pi^{-1} \mathscr{D}_{s}\right) \rightarrow \pi_{*} \mathscr{C}_{0}^{R, s} \rightarrow \\
\quad \rightarrow R^{1} \pi_{*} \underline{\Gamma}_{s^{*}}\left(\pi^{-1} \mathscr{D}_{s}\right) \rightarrow R^{1} \pi_{*} \underline{\Gamma}_{s^{*}}\left(\pi^{-1} \mathscr{D}_{s}\right) \rightarrow \cdots
\end{aligned}
$$

Il nous reste à calculer $R^{i} \pi_{*} \underline{\Gamma}_{s^{*}}\left(\pi^{-1} \mathscr{D}_{s}\right)$ pour $i=0,1$.

D'après la proposition V. 1 , il suffit de calculer $R^{i} \tau_{*} \underline{\Gamma}_{s}\left(\tau^{-1}{ }^{\prime} \mathscr{D}_{s}\right)$, ce qui est plus simple car l'application $\tau$ est propre et séparée.

*) Calcul de $\tau_{*} \underline{\Gamma}_{s}\left(\tau^{-1}{ }^{\prime} \mathscr{D}_{s}\right)$.

Ecrivons la suite exacte de cohomologie locale de $\tau^{-1} \mathscr{D}_{s}$ à support dans $S$

$$
0 \rightarrow \underline{\Gamma}_{s}\left(\tau^{-1} \mathscr{D}_{s}\right) \rightarrow \underline{\Gamma}\left(\tilde{C}, \tau^{-1} \mathscr{D}_{s}\right) \rightarrow \underline{\Gamma}\left(\tilde{C}-S, \tau^{-1} \mathscr{D}_{s}\right) \rightarrow \cdots
$$

Comme $\underline{\Gamma}_{S}\left(\tau^{-1} \mathscr{D}_{s}\right)$ est concentré sur $S$, on s'intéresse à la fibre en $(0, \xi)$ de cette suite exacte, mais sur $S, \tau^{-1}{ }^{\prime} \mathscr{D}_{s}$ est le faisceau constant de fibre $\left(\mathscr{D}_{s}\right)_{0}$ et de plus, $\underline{\Gamma}\left(\tilde{C}-S, \tau^{-1}{ }^{\prime} \mathscr{D}_{s}\right)=\underline{\Gamma}\left(\boldsymbol{C}-\{0\}, \mathscr{D}_{s}\right)$. Donc $\underline{\Gamma}_{S}\left(\tau^{-1} \mathscr{D}_{s}\right)$ est le faisceau constant sur $S$ dont la fibre est le noyau de la restriction:

$$
\left(\mathscr{D}_{s}\right)_{0} \rightarrow\left(\underline{\Gamma}\left(\boldsymbol{C}-\{0\}, \mathscr{D}_{s}\right)\right)_{0}
$$

c'est-à-dire $\left(\underline{\Gamma}_{10\}}\left(\mathscr{D}_{s}\right)\right)_{0}$.

On a donc $\left(\tau_{*} \underline{\Gamma}_{S}\left(\tau^{-1} \mathscr{D}_{s}\right)\right)=\Gamma\left(S, \underline{\Gamma}_{S}\left(\tau^{-1} \mathscr{D}_{s}\right)\right)$ puisque $\tau$ est propre et séparée ([2]), donc 


$$
\tau_{*} \underline{\Gamma}_{S}\left(\tau^{-1^{\prime} \mathscr{D}_{s}}\right)=\Gamma_{\{0\}}\left(\mathscr{D}_{s}\right) .
$$

*) Calcul de $R^{1} \underline{\Gamma}_{S}\left(\tau^{-1} \mathscr{D}_{s}\right)$.

Le théorème de Whitney fournit la suite exacte de faisceaux

$$
0 \rightarrow \underline{\Gamma}_{(0)}\left(\mathscr{D}_{s}\right) \rightarrow^{\prime} \mathscr{D}_{s} \rightarrow^{\prime} \mathscr{S}_{s,(0)} \rightarrow 0 .
$$

On en déduit d'après le calcul précédent et l'exactitude du foncteur $\tau^{-1}$ une autre suite exacte:

$$
0 \rightarrow \underline{\Gamma}_{S}\left(\tau^{-1^{\prime}} \mathscr{D}_{s}\right) \rightarrow \tau^{-1^{\prime}} \mathscr{D}_{s} \rightarrow \tau^{-1^{\prime}} \mathscr{S}_{s,(0)} \rightarrow 0 .
$$

En appliquant le foncteur $\tau_{*}$ on obtient une longue suite exacte

$$
\begin{aligned}
0 & \rightarrow \tau_{*} \underline{\Gamma}_{S}\left(\tau^{-1^{\prime}} \mathscr{D}_{s}\right) \rightarrow \tau_{*} \tau^{-1^{\prime}} \mathscr{D}_{s} \rightarrow \tau_{*} \tau^{-1^{\prime}} \mathscr{S}_{s,\{0\}} \rightarrow R^{1} \tau_{*} \underline{\Gamma}_{S}\left(\tau^{-1^{\prime}} \mathscr{D}_{s}\right) \rightarrow \\
& \rightarrow R^{1} \tau_{*} \tau^{-1^{\prime}} \mathscr{D}_{s} \rightarrow R^{1} \tau_{*} \tau^{-1^{\prime}} \mathscr{S}_{s,\{0\}} \rightarrow \cdots .
\end{aligned}
$$

On a (cf. [2] ch. IV par ex.)

$$
\begin{aligned}
& \tau_{*} \tau^{-1^{\prime}} \mathscr{D}_{s}=\mathscr{D}_{s} \quad R^{1} \tau_{*} \tau^{-1^{\prime}} \mathscr{D}_{s 1 S}=\left({ }^{\prime} \mathscr{D}_{s}\right)_{0} \quad R^{1} \tau_{*} \tau^{-1^{\prime}} \mathscr{D}_{s \mid \tilde{C}-s}=0 \\
& \tau_{*} \tau^{-1^{\prime}} \mathscr{S}_{s,\{0\}}={ }^{\prime} \mathscr{S}_{s,\{0\}} \quad R^{1} \tau_{*} \tau^{-1^{\prime}} \mathscr{S}_{s,\{0\} \mid S}=\left({ }^{\prime} \mathscr{S}_{s,(0)}\right)_{0} \\
& R^{1} \tau_{*} \tau^{-1^{\prime}} \mathscr{S}_{s,(0) \mid \tilde{c}-S}=0
\end{aligned}
$$

(les foncteurs dérivés $R^{i}$ pour $\mathrm{i}>1$ sont nuls).

Comme $\tau_{*} \underline{\Gamma}_{S}\left(\tau^{-1^{\prime}} \mathscr{D}_{s}\right)=\underline{\Gamma}_{\{0\}}\left(\mathscr{D}_{s}\right)$, en scindant la suite exacte cidessus en suite exacte courte, on obtient la suite exacte:

$$
0 \rightarrow \mathscr{S}_{s,\{0\}} \rightarrow R^{1} \tau_{*} \underline{\Gamma}_{S}\left(\tau^{-1^{\prime}} \mathscr{D}_{0}\right) \rightarrow \underline{\Gamma}_{\{0\}}\left(\mathscr{D}_{s}\right) \rightarrow 0
$$

d'où:

$$
R^{1} \tau_{*} \underline{\Gamma}_{S}\left(\tau^{-1^{\prime}} \mathscr{D}_{s}\right)=^{\prime} \mathscr{D}_{s}
$$

*) Calcul final.

On a la suite exacte longue:

$$
0 \rightarrow \Gamma_{\{0\}}\left({ }^{\prime} \mathscr{D}_{s}\right) \stackrel{\dot{\partial}}{\longrightarrow} \Gamma_{\{0\}}\left({ }^{\prime} \mathscr{D}_{s}\right) \rightarrow\left(\pi_{*} \mathscr{C}_{0}^{R, s}\right)_{0} \rightarrow\left({ }^{\prime} \mathscr{D}_{s}\right)_{0} \stackrel{\dot{\partial}}{\longrightarrow}\left({ }^{\prime} \mathscr{D}_{s}\right)_{0} \rightarrow \cdots .
$$

En scindant celle-ci en une suite exacte courte, on obtient une suite exacte:

$$
0 \rightarrow \frac{\Gamma_{\{0\}}\left(\mathscr{D}_{s}\right)}{\bar{\partial} \Gamma_{[0\}}\left({ }^{\prime} \mathscr{D}_{s}\right)} \rightarrow\left(\pi_{*} \mathscr{C}_{0}^{R, s}\right)_{0} \rightarrow \mathcal{O}_{c \mid\{0\}} \rightarrow 0
$$

et grâce au lemme IV. 7 on a:

$$
\mathscr{U}_{s}=\frac{\Gamma_{(0)}\left({ }^{\prime} \mathscr{D}_{s}\right)}{\partial \Gamma{ }^{\prime 01}\left(\mathscr{D}_{s}\right)}
$$


ce qui termine la démonstration.

Remarques. 1) Pour $s=1, \mathscr{C}_{s}$ désigne les opérateurs différentiels d'ordre infini à coefficients constants, et comme ' $\mathscr{D}_{1}$ désigne le faisceau des hyperfonctions, qui est un faisceau flasque, $\mathscr{U}_{1}=\underline{H}_{\{0\}}^{1}(\mathcal{O})$ on retrouve la suite exacte de [18]

$$
0 \rightarrow \underline{H}_{\{0\}}^{1}(\mathcal{O}) \rightarrow \pi_{*} \mathscr{C}_{0}^{\boldsymbol{R}} \rightarrow \mathcal{O}_{c \mid\{0\}} \rightarrow 0
$$

2) on peut montrer qu'on a un isomorphisme d'espaces vectoriels (sans topologie)

$$
\left(R^{1} \tau_{*} \mathscr{C}_{0, s}\right)_{0}=H^{1}\left(S, \mathscr{A}_{0, s}\right)=H_{c}^{1}\left(\tilde{\boldsymbol{C}}, \mathscr{A}_{o, s}\right)=\frac{\boldsymbol{C}[[z]]_{s}}{\boldsymbol{C}\{z\}} .
$$

Remarquons aussi, puisque $\mathscr{C}_{0}^{\boldsymbol{R}, s}$ est un faisceau concentré sur $S^{*}$ qu'on a un isomorphisme d'espaces vectoriels:

$$
\Gamma\left(\tilde{\boldsymbol{C}}^{*}, \mathscr{C}_{0}^{\boldsymbol{R}, s}\right)=\Gamma\left(S^{*}, \mathscr{C}_{0}^{\boldsymbol{R}, s}\right) .
$$

$4^{\circ}$ ) Représentation des microfonctions.

Notons $B_{\varepsilon}$ la boule de centre 0 et de rayon $\varepsilon$ dans $C$. Si $\xi=e^{i \theta}$, notons $V_{\varepsilon, \zeta}$ l'intersection du secteur fermé

$$
\{z|| \operatorname{Arg} z-\theta \mid \leq \pi / 2+\varepsilon\} \text { avec } B_{\varepsilon} \text {. }
$$

Proposition V.5. $\quad\left(\mathscr{C}_{0}^{R, s}\right)_{(0, \zeta)}=\underset{\varepsilon}{\lim } \frac{\mathcal{O}_{t \cdot \bmod }\left(B_{\varepsilon}-V_{\varepsilon, \zeta}\right)}{\mathcal{O}\left(B_{\varepsilon}\right)}$ où $t=\frac{1}{s-1}$

Démonstration. On a une suite exacte

$$
0 \rightarrow \underline{\Gamma}_{s^{*}}\left(\pi^{-1} \mathscr{D}_{s}\right) \rightarrow \underline{\Gamma}_{s^{*}}\left(\pi^{-1} \mathscr{D}_{s}\right) \rightarrow \mathscr{C}_{0}^{\boldsymbol{R}, s} \rightarrow 0
$$

et grâce à la proposition V.2:

$$
\left(\underline{\Gamma}_{s^{*}}\left(\pi^{-1} \mathscr{D}_{s}\right)\right)_{(0, \zeta)}=\underset{\varepsilon}{\lim } \Gamma_{V_{\varepsilon, \zeta}}\left(B_{\varepsilon}, \mathscr{D}_{s}\right) .
$$

De plus par la proposition III. 2, on a

$$
\mathcal{O}_{t . \bmod }\left(B_{\varepsilon}-V_{\varepsilon, \zeta}\right)=\operatorname{Ker}\left({ }^{\prime} \mathscr{S}_{V_{\varepsilon, \zeta}} \stackrel{\bar{\partial}}{\longrightarrow} \mathscr{S}_{V_{s, \zeta}}\right) \text {. }
$$

En écrivant la longue suite exacte associée à la suite exacte de complexes donnée par le théorème de Whitney dual on obtient le résultat. 
$\left.5^{\circ}\right)$ Un théorème d'indice dans les microfonctions.

Faisons tout d'abord un rappel sur l'irrégularité de Katz d'un opérateur différentiel ([3] par ex). Soit $P=\sum_{0 \leq i \leq n} a_{i}(z) \partial^{i}$ un opérateur différentiel d'ordre fini à coefficients analytiques au voisinage de $0\left(\partial=\frac{d}{d z}\right)$. En développant chaque $a_{i}(z)$ en série on obtient

$$
P=\sum_{\substack{0 \leq j<+\infty \\ 0 \leq i \leq m}} a_{i, j} z^{j} \partial^{i}
$$

on note $N(P)=\left\{(i, j-i) \in \mathbb{R}^{2} a_{i, j} \neq 0\right\}$ et $\mathscr{P}(D)$ l'enveloppe convexe de $N(P)$ s'appelle le polygône de Newton de $P$. (On ne considère dans ce polynôme que les droites de pente positive).

L'invariant de Katz, ou irrégularité de Katz de $P$ est la plus grande pente, notée $k(P)$, de ce polygône.

On note $\mathscr{C}_{0}^{\boldsymbol{R}, s, P}=\operatorname{Ker}\left(\mathscr{C}_{0}^{\boldsymbol{R}, s} \stackrel{P}{\longrightarrow} \mathscr{C}_{0}^{\boldsymbol{R}, s}\right) t=\frac{1}{s-1}$.

Theoreme V.6. Si $t>k(P)$ on a une suite exacte

$$
0 \longrightarrow \mathscr{C}_{0}^{\boldsymbol{R}, s, P} \longrightarrow \mathscr{C}_{0}^{\boldsymbol{R}, s} \stackrel{P}{\longrightarrow} \mathscr{C}_{0}^{\boldsymbol{R}, s} \longrightarrow 0 .
$$

De plus $\mathscr{C}_{0}^{R, s, P}$ est de dimension finie égale à $\operatorname{val}\left(a_{m}\right)$.

Démonstration. D'après [7], [12] le résultat est vrai pour $s=1$ c'est-à-dire pour $\mathscr{C}_{0}^{\boldsymbol{R}}$.

Il reste donc à montrer que si $\bar{f} \in \mathscr{C}_{0}^{R, s}$ et $\bar{u} \in \mathscr{C}_{0}^{R}$ vérifiant $P \bar{u}=\bar{f}$ alors $\bar{u} \in \mathscr{C}_{0}^{\boldsymbol{R}, s}$. Notons $u$ un représentant de $\bar{u}$ dans $\mathcal{O}\left(B_{\varepsilon}-V_{\varepsilon, \zeta}\right)$ et $f$ un représentant $\bar{f}$ dans $\mathcal{O}_{t \cdot \bmod }\left(B_{\varepsilon}-V_{\varepsilon, \zeta}\right)$

$$
P=\sum a_{i, j} z^{j-(t+1) i} z^{(t+1) i} \partial^{i}
$$

D'après l'hypothèse sur $t j-(t+1) i \geq \operatorname{val}\left(a_{m}\right)-(t+1) m$. Ainsi quitte à

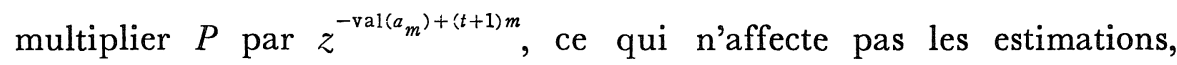
on peut supposer que

$$
P=z^{(t+1) m} \partial^{m}+\sum_{0 \leq i \leq m-1} c_{i}(z)\left[z^{(t+1) i} \partial^{i}\right]
$$

ce qui s'écrit [9] 


$$
P=\left(z^{t+1} \partial\right)^{m}+\sum_{0 \leq i \leq m-1} z^{\alpha_{i}} b_{i}(z)\left(z^{t+1} \partial\right)^{i}
$$

où $\alpha_{i}>0$ et $b_{i}(z)$ est holomorphe et ne s'annule pas au voisinage de zéro.

Soit $Z=\left(\frac{1}{z}\right)^{t}$, par ce changement de coordonnées $P$ se transforme en (nonobstant les constantes)

$$
P=\left(\frac{d}{d z}\right)^{m}+\sum_{0 \leq i \leq m-1}\left(\frac{1}{Z^{t}}\right)^{\alpha_{i}} a_{i}\left(\frac{1}{Z^{1 / t}}\right)\left(\frac{d}{d Z}\right)^{i} .
$$

Si pour simplifier on prend par exemple $\zeta=i$

$$
B_{\varepsilon}-Z_{\varepsilon, i}=\{|z|<\varepsilon \quad-\varepsilon<\operatorname{Arg} z<\pi+\varepsilon\} .
$$

Se transforme en un secteur à l'infini

$$
D_{\varepsilon, i}=\left\{|Z|>\left(\frac{1}{\varepsilon}\right)^{t} \quad-t(\pi+\varepsilon)<\operatorname{Arg} Z<t \varepsilon\right\}
$$

$\mathcal{O}_{t . \bmod }\left(B_{\varepsilon}-Z_{\varepsilon, i}\right)$ se transforme en l'espace des fonctions holomorphes de type exponentiel nul sur $D_{\varepsilon, i}$ qu'on notera $\mathcal{O}_{-}\left(D_{\varepsilon, i}\right)$.

Soit $W$ un sous-secteur à l'infini de $D_{\varepsilon, i}$ on note $L_{\eta, W}$ l'espace de Banach des fonctions de $\mathcal{O}\left(D_{\varepsilon, i}\right)$ telles que

$$
\|f\|_{\eta, W}=\operatorname{Sup}_{W}\left|f(z) e^{-\eta|z|}\right|<+\infty .
$$

On munit $\mathcal{O}_{-}\left(D_{\varepsilon, i}\right)$ de la topologie définie par ces semi-normes:

$$
\mathcal{O}_{-}\left(D_{\varepsilon, i}\right)=\lim _{\overleftarrow{W, \eta}} L_{\eta, W}
$$

Soit $\mathrm{a} \in D_{\varepsilon, i}$ on note

$$
\partial_{a}^{-1} f(z)=\int_{a}^{z} f(t) d t
$$

Montrons que $\partial_{a}^{-1}$ est un opérateur linéaire continu de $L_{\eta, W}$ dans $L_{\eta, W}$ : Soit $t=s(z-a)+a s \in[0,1]$ a étant bien choisi

$$
\begin{aligned}
\left|\partial_{a}^{-1} f(z)\right|= & \left|\int_{0}^{1} f(s(z-a)+a)(z-a) d s\right| \leq|z-a| e^{\eta|a|}|| f \|_{\eta, W} \\
& \int_{0}^{1} e^{\eta s|z-a|} d s \leq e^{2 \eta|a|} e^{\eta|z|}\|f\|_{\eta, W}
\end{aligned}
$$

Il suffit alors de montrer le résultat pour

$$
P_{0}=1+\sum_{0 \leq i \leq m-1}\left(\frac{1}{Z^{t}}\right)^{\alpha_{i}} a_{i}\left(\frac{1}{Z^{1 / t}}\right) \partial^{i-m} .
$$

Soit en changeant de notation 


$$
P_{0}=1+\sum_{1 \leq i \leq m}\left(\frac{1}{Z}\right)^{\beta_{i}} b_{i}(Z) \partial^{-i}=1+P_{1}
$$

Pour $\varepsilon$ assez grand $\left\|P_{1}\right\|_{\eta, W}<1$ donc $P_{0}=1+P_{1}$ réalise une bijection de $L_{\eta, W}$ sur lui-même on en déduit le résultat.

Remarque. En reprenant l'argument de [9] on peut construire une solution $\bar{u}$ de $\mathscr{C}_{0}^{R}$ vérifiant $P \bar{u}=0$ qui n'appartient pas à $\mathscr{C}_{0}^{\boldsymbol{R}, s}$ pour tout $s$ vérifiant $t<k(P)$. Donc dans le cas $t<k(P)$ si $\mathscr{C}_{0}^{R, s, P}$ est toujours de dimension finie, il est différent de $\mathscr{C}_{0}^{\boldsymbol{R}, P}$.

$6^{\circ}$ ) Application à l'étude des équations à points singuliers irréguliers.

On retrouve un théorème de [16]:

Theoreme V.7. Soit $P$ un opérateur différentiel d'ordre fini à coefficients analytiques, soit $s$ tel que $t=\frac{1}{s-1}>k(p)$. On a un quasiisomorphisme

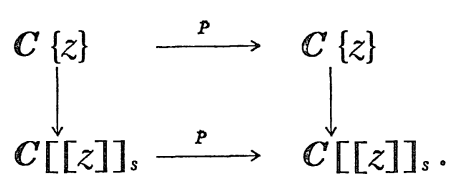

Démonstration. $U_{s}$ étant en dualité avec $\mathbb{C}[[z]]_{s}$, il suffit de montrer (cf. [16]) le quasi isomorphisme suivant

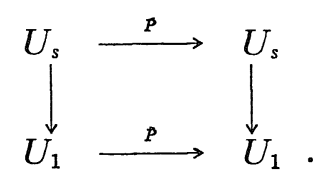

D'après le théorème $\mathrm{V} \cdot 6$, on a un quasi-isomorphisme

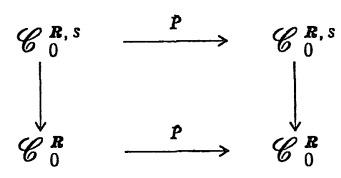

en appliquant $\pi_{*}$, par le théorème $\mathrm{V} .4$ on obtient un autre quasiisomorphisme 


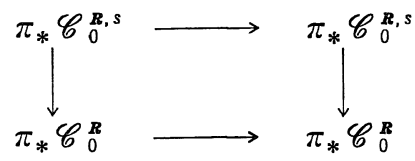

Notons $U_{s}^{*}$ le complexe $U_{s} \stackrel{P}{\longrightarrow} U_{s}$

$K_{s}^{*}$ le complexe $\pi_{*} \mathscr{C}^{R, s} \stackrel{P}{\longrightarrow} \pi_{*} \mathscr{C}^{R, s}$

$L \cdot$ le complexe $\mathcal{O}_{c \mid\{0\}} \stackrel{P}{\longrightarrow} \mathcal{O}_{\boldsymbol{c} \mid\{0\}}$

Par le théorème V.4, on a un morphisme entre suites exactes de complexes:

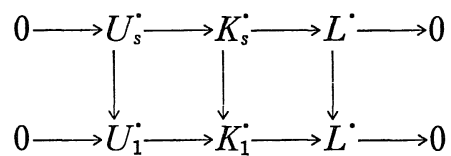

En appliquant les longues suites exactes de cohomologie on obtient un morphisme de suites exactes

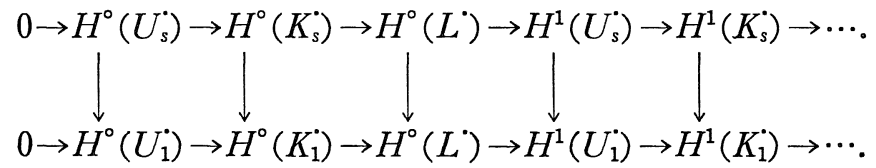

Le résultat précédent et le lemme des cinq terminent alors la démonstration.

\section{Bibliographie}

[1] A. Andreotti, C. Banica, Relative duality on complex spaces, Revue Roum. de Math. 9 (1975, 1976).

[2] G.E. Bredon, Sheaf Theory, McGraw hill, New-York 1966.

[3] P. Deligne, Equations différentielles à points singuliers réguliers, Springer Lectures Notes 163, Springer-Verlag Berlin and New-York, 1970.

[4] I. M. Guelfand, G. E. Chilov, Les distributions, Dunod Paris 1964.

[5] A. Grothendieck, Espaces vectoriels topologiques Gordon and Breach 1973.

[6] J. M. Kantor, Classes non quasi analytiques et décomposition des supports des ultradistributions, An. Acad. Brasil Ciencas 44.2(1972), 171-180.

[7] M. Kashiwara, Systèmes d'équations microdifférentielles, Paris-Nord, 1977.

[8] H. Komatsu, Ultradistributions I et II, J. Fac. Univ. Tokyo Sect IA 20 (1973), 25-105, Sect. IA 24 (1977), 607-628.

[9] On the regularity of hyperfunction solutions of linear ordinary differential equations with real coefficients, J. Fac. Univ. Tokyo Sect. A20 (1973).

[10] B. Malgrange, Remarques sur les équations différentielles à points singuliers irréguliers, Lecture Notes in Math. 712, Springer-Verlag, 1979.

[11] $\longrightarrow$ Sur les points singuliers des équations différentielles, Ens. Math. 20 (1970), 147-176. 
[12] B. Malgrange, Rapport sur les théorèmes d'indice de Boutet de Monvel et Kashiwara, Preprint Université de Grenoble.

[13] Modules microdifferentiels et classes de Gevrey, Mathematical analysis and applications Part B., Advances in Math. Supplementary studies 7B, Academic Press, 1981.

[14] F. Pham, Introduction a l'étude des systèmes différentiels de Gauss-Manin" Birkauser, 1980.

[15] J. P. Ramis, Devissage Gevrey, Astérique S.M.F. 59-60 (1978), 173-204.

[16] Théorèmes d'indice Gevrey pour les équations différentielles ordinaires, (Prépublication IRMA Strasbourg 1981), A paraître aux "Memoirs of the A.M.S."

[17] Les séries $k$-sommables et leurs applications, Springer Lecture Notes in Physics, 126 Springer-Verlag, 1980.

[18] M. Sato, T. Kawai, M. Kashiwara, Microfunctions and pseudodifferential equations, Springer Lecture Notes 287 (1973), 264-529, Springer-Verlag.

[19] I. Cioranescu, Operator-Valued Ultradistributions in the Spectral Theory, Math. Ann. 223 (1976), 1-12.

[20] B. Droste, Extension of analytic functional calculus mappings and duality by $\bar{\partial}-$ closed forms with grow, Math. Ann. 261 (1982), 185-200.

[21] De Roever, Complex fourier transform and analytical functionals with unbounded Carriers" Math. Centre Tracts 89 Amsterdam, 1978. 
